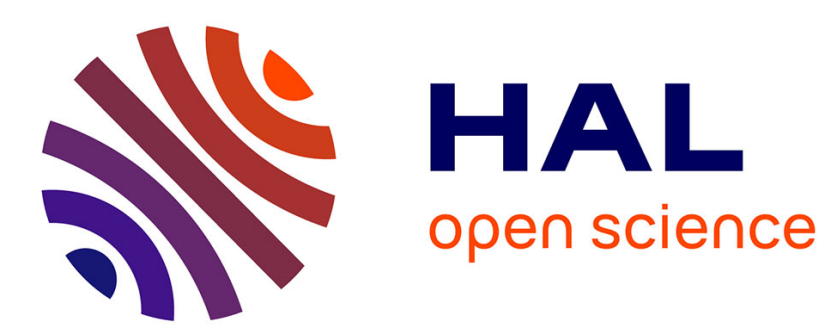

\title{
LiveRank: How to Refresh Old Crawls
}

\author{
The Dang Huynh, Fabien Mathieu, Laurent Viennot
}

\section{To cite this version:}

The Dang Huynh, Fabien Mathieu, Laurent Viennot. LiveRank: How to Refresh Old Crawls. Algorithms and Models for the Web Graph - 11th International Workshop (WAW 2014), Dec 2014, Beijing, China. pp.148 - 160, 10.1007/978-3-319-13123-8_12 . hal-01093188

\section{HAL Id: hal-01093188 \\ https://hal.inria.fr/hal-01093188}

Submitted on 10 Dec 2014

HAL is a multi-disciplinary open access archive for the deposit and dissemination of scientific research documents, whether they are published or not. The documents may come from teaching and research institutions in France or abroad, or from public or private research centers.
L'archive ouverte pluridisciplinaire HAL, est destinée au dépôt et à la diffusion de documents scientifiques de niveau recherche, publiés ou non, émanant des établissements d'enseignement et de recherche français ou étrangers, des laboratoires publics ou privés. 


\title{
LiveRank: How to Refresh Old Crawls
}

\author{
The Dang Huynh ${ }^{1,2}$, Fabien Mathieu ${ }^{1}$, and Laurent Viennot ${ }^{2}$ \\ 1 Alcatel-Lucent Bell Labs France \\ 2 Inria - Univ. Paris Diderot
}

\begin{abstract}
This paper considers the problem of refreshing a crawl. More precisely, given a collection of Web pages (with hyperlinks) gathered at some time, we want to identify a significant fraction of these pages that still exist at present time. The liveness of an old page can be tested through an online query at present time. We call LiveRank a ranking of the old pages so that active nodes are more likely to appear first. The quality of a LiveRank is measured by the number of queries necessary to identify a given fraction of the alive pages when using the LiveRank order. We study different scenarios from a static setting where the LiveRank is computed before any query is made, to dynamic settings where the LiveRank can be updated as queries are processed. Our results show that building on the PageRank can lead to efficient LiveRanks for Web graphs.
\end{abstract}

\section{Introduction}

One of the main challenges for large networks data mining is to deal with the high dynamics of huge datasets: not only are these datasets difficult to gather, but they tend to become obsolete very quickly.

In this paper, we are interested in the evolution of large Web graphs at large time scale. We focus on batch crawling, where starting from a completely outdated snapshot of a large Web crawl, we want to identify a significant fraction of the pages that are still alive now.

Our motivation is that many old large snapshots of the Web are available today. Reconstructing roughly what remains from such archives could result in interesting studies of the long term evolution of these graphs. For large archives where one is interested in a fraction of the dataset, recrawling the full set of pages can be prohibitive. We propose to identify as quickly as possible a significant fraction of the pages that are still alive. Further selection can then be made to identify a set of pages suitable for the study and then to crawl them. Such techniques would be especially interesting when testing the liveness of an item is much lighter than downloading it completely. This is for instance the case for the Web with HEAD queries compared to GET queries. If a large amount of work has been devoted to maintaining fresh a set of crawled pages, little attention has been paid to the coverage obtained by partial recrawling a fairly old snapshot.

Problem formulation: Given an old snapshot, our goal is to identify a significant fraction of the pages that are still alive or active now. The cost we incur is the number of fetches that are necessary to attain this goal. A typical cost 
measure will be the average number of fetches per active item identified. The strategy for achieving this goal consists in producing an ordering for fetching the pages. We call LiveRank an ordering such that the pages that are still alive tend to appear first. We consider the problem of finding an efficient LiveRank in three settings: static when it is computed solely from the snapshot and the link relations recorded at that time; sampling-based when a sampling is performed in a first phase allowing to adjust the ordering according to the liveness of sampled items; dynamic when it is incrementally computed as pages are fetched.

Contribution: We propose various LiveRank algorithms based on the graph structure of the snapshot. We evaluate them on two Web snapshots (from 10 to 20 million nodes). We show that a rather simple combination of a small sampling phase and PageRank-like propagation in the remaining of the snapshot allows to gather from $15 \%$ to $75 \%$ of the active nodes with a cost that remains within a factor of 2 from the optimal ideal solution.

Related work: The process of crawling the Web has been extensively studied. A survey is given by Olston and Najork [12].

The issue we investigate here is close to a problem introduced by Cho and Ntoulas [6]: they use sampling to estimate the frequency of change per site and then to fetch a set of pages such that the overall change ratio of the set is maximized. Their technique consists in estimating the frequency of page change per site and to crawl first sites with high frequency change. Tan et al. [15] improve slightly over this technique by clusterizing the pages according to several features: not only their site (and other features read from the URL) but also content based features and linkage features (including pagerank and incoming degree). A change ratio per cluster is then estimated through sampling and clusters are downloaded in descending order of the estimated values. More recently, Radinsky and Bennett [14] investigate a similar approach using learning techniques and avoiding the use of sampling.

Note that these approaches mainly focus on highly dynamic pages and use various information about pages whereas we are interested in stable pages and we use only the graph structure, which is lighter.

With a slightly different objective, Dasgupta et al. [7] investigate how to discover new pages while minimizing the average number of fetches per new page found. Their work advocates for: a greedy cover heuristic when a small fraction of the new pages has to be discovered quickly: an out-degree-based heuristic gathering a large fraction of the new pages. Their framework is close to ours and inspired the cost function used in this paper.

A related problem consist in estimating which pages are really valid among the "dangling" pages on the frontier of the crawled web (those that are pointed by crawled pages but that were not crawled themselves). Eiron et al. propose to take this into account in the PageRank computation [8]. In a similar trend, BarYossef et al. [2] propose to compute a "decay" score for each page by refining on the proportion of dead links in a page. Their goal is to identify poorly updated pages. This score could be an interesting measure for computing a LiveRank, 
however its computation requires to identify dead links. It is thus not clear how to both estimate it and at the same time try to avoid to test dead pages.

Roadmap: In the next Section, we propose a simple cost function to evaluate the quality of a LiveRank and we introduce several classes of possible LiveRanks. For evaluating the proposals, we use two datasets from the . uk Web, for which we derived some ground truth of page liveness. This is described in Section 3. Lastly, in Section 4, we benchmark our LiveRanks against the datasets and discuss the results.

\section{Model}

Let $G=(V, E)$ be a directed graph obtained from a past Web snapshot, where $V$ represents the crawled pages and $E$ the hyperlinks: For $i, j$ in $V,(i, j)$ is in $E$ if, and only if, there is a hyperlink to $j$ in $i$.

Let $n$ denote the size of $V$. At present time, only a subset of $G$ are still alive (the exact meaning of liveness will be detailed in the next Section). We call $a$ the function that tells if pages are alive or not: $a(X)$ denotes the alive pages from $X \subset V$, while $\bar{a}(X)$ stands for $X \backslash a(X)$. Let $n_{a}$ be $|a(V)|$.

The problem we need to solve can be expressed as: how to crawl a maximum number of pages from $a(V)$ with a minimal crawling cost. In particular, one would like to avoid crawling too many pages from $\bar{a}(V)$. If $a$ was known, the task would be easy, but testing the activity of a node obviously requires to crawl it. This is the rationale for the notion of LiveRank.

\subsection{Performance metric}

Formally, any ordering on $V$ can be seen as a LiveRank, so we need some performance metrics to measure the efficiency in ranking the pages from $a(V)$ first. Following [7], we define the LiveRank cost as the average number of page retrievals necessary to obtain one alive page, after a fraction $0<\alpha \leq 1$ of the alive pages has been retrieved.

In details, let $\mathcal{L}_{i}$ represent the $i$ first pages returned by a LiveRank $\mathcal{L}$, and let $i(\mathcal{L}, \alpha)$ be the smallest integer such that $\frac{\left|a\left(\mathcal{L}_{i}\right)\right|}{n_{a}} \geq \alpha$. The cost function of $\mathcal{L}$ (which depends on $\alpha$ ) is then defined by:

A few remarks on the cost function:

$$
\operatorname{cost}(\mathcal{L}, \alpha)=\frac{i(\mathcal{L}, \alpha)}{\alpha n_{a}} .
$$

- It is always at greater than or equal to 1 . An ideal LiveRank would perfectly separate $a(V)$ from rest of the nodes, so its cost function would be 1 . Without some oracle, this requires to test all pages, which is exactly what we would like to avoid. The cost function allows to capture this dilemma.

- Keeping a low cost becomes hard as $\alpha$ gets close to 1: without some clairvoyant knowledge, capturing almost all active nodes is almost as difficult as capturing all actives nodes. For that reason, one expects that when $\alpha$ gets close to 1 , the set of nodes any real LiveRank will need to crawl will tend to $V$, leading to an asymptotical cost $\frac{n}{n_{a}}$. This will be verified in Section 4 . 
- Lastly, one may have noticed that the cost function uses $n_{a}=|a(V)|$, for which an exact value requires a full knowledge of liveness. This is not an issue here as we will perform our evaluation on datasets where $a$ is known. For use on datasets without ground truth, one could either use an estimation of $n_{a}$ based on a sampling or use a non-normalized cost function (for instance the fraction of alive pages obtained after $i$ retrievals).

\subsection{PageRank}

Some of the proposed LiveRanks are based on PageRank. In order to be selfcontained, we provide a brief reminder. PageRank is a link analysis algorithm that has been initially introduced in [13] and used by the Google Internet search engine. It assigns a numerical importance to each page of a Web graph. The principle of PageRank [13] is to use the structural information from $G$ to attribute importance according to the following (informal) recursive definition: $a$ page is important if it is referenced by important pages. Concretely, to compute PageRank value, denoted by the row vector $Y$, one needs to find the solution of the following equation:

$$
Y=d Y A+(1-d) X
$$

where $A$ is a substochastic matrix derived from the adjacency matrix of $G$, $d<1$ a so-called damping factor (often set empirically to $d=0.85$ ), and $X \supsetneqq 0$ is a teleportation vector. $X$ represents a kind of importance by default that is propagated from pages to pages according to $A$ with a damping $d$.

Computation of PageRank vectors has being widely studied. Several specific solutions were proposed and analysed [11,3] including power method [13], extrapolation $[9,10]$, adaptive on-line method [1], etc.

We now present the different LiveRanks that we will consider in this paper. We broadly classify them in three classes: static, sample-based and dynamic.

\subsection{Static LiveRanks}

Static LiveRanks are computed offline using uniquely the information from $G$. That makes them very basic, but also very easy to be used in a distributed way: given $p$ crawlers of similar capacities, if $\mathcal{L}=\left(l_{1}, \ldots, l_{n}\right)$, simply assign the task of testing node $l_{i}$ to crawler $i \bmod p$.

We propose the following three static LiveRanks.

Random permutation $(R)$ will serve both as a reference and as a building block for more advanced LiveRanks. $R$ ignores any information from $G$, so its cost should be in average $\frac{n}{n_{a}}$, with a variance that tends to 0 as $\alpha$ tends to 1 . We expect good LiveRanks to have a cost function significantly lower than $\operatorname{cost}(R)$.

Decreasing Indegree ordering (I) is a simple LiveRank that we expect to behave better than a random permutation. Intuitively, a high Indegree can mean some importance, and important pages may be more robust. Also, older pages should have more incoming edges (in terms of correlation), so high degree pages can correspond to pages that were already old at the time $G$ was crawled, and old pages may last longer than younger ones. Sorting by degree is the easiest way to exploit these correlations. 
PageRank ordering $(P)$ pushes forward the indegree idea. The intuition is that pages that are still alive are likely to point toward pages that are still alive also, even considering only old links. This suggests to use a PageRank-like importance ranking. In absence of further knowledge, we propose to use the solution of (1) using $d=.85$ (typical value for Web graphs) and $X$ uniform on $V$.

Note that it is very subjective to evaluate PageRank as an importance ranking, as importance should be ultimately validated by humans. On the other hand, the quality of PageRank as a static LiveRank is straightforward to verify, for instance using our cost metric.

The possible existence of correlation between Indegree (or PageRank) and liveness will be verified in Section 3.3.

\subsection{Sample-based LiveRanks}

Using a LiveRank consists in crawling $V$ in the prescribed order. During the crawl, the activity function $a$ becomes partly available, and it is natural to reinject the obtained information to enhance the retrieval, producing a new LiveRank. Following that idea, we consider here a two-steps sample-based approach: we first fix a testing threshold $z$ and test $z$ items following a static LiveRank (like $R, I$ or $P$ ). For the set $Z$ of nodes tested, which we call indifferently sample set or training set, we thus obtain the knowledge of $a(Z)$ and $\bar{a}(Z)$, which allows us to recompute the LiveRank of the remaining untested pages.

Because the sampling uses a static LiveRank, and the adjusted new LiveRank is static as well, sample-based LiveRanks are still easy to use in a distributed way as the crawlers only need to receive crawl instructions on two occasions.

Notice that in the case where the sampling LiveRank is a random permutation, $|a(Z)| \frac{n}{z}$ can be used as an estimate for $n_{a}$. This can for instance be used to decide when to stop crawling if we desire to identify $\alpha n_{a}$ active nodes in $a(V)$.

Simple adaptive LiveRank $\left(P_{a}\right)$ When a page is alive, we can assume it increases the chance that pages it points to in $G$ are also alive, and that life is transmitted somehow through hyperlinks. Following this idea, a possible adaptive LiveRank consists in taking for $X$ in (1) the uniform distribution on $a(Z)$. This diffusion from such an initial set can be seen as a kind of breadth-first traversal starting from $a(Z)$, but with a PageRank flavour.

Double adaptive LiveRank $\left(P_{a}^{+/-}\right)$The simple adaptive LiveRank does not use the information given by $\bar{a}(Z)$. One way to do this is to calculate an "anti"PageRank based on $\bar{a}(Z)$ instead of $a(Z)$. This ranking would represents a kind of diffusion of death, the underlying hypothesis being that dead pages may point to pages that tend to be dead. As a result, we obtain a new LiveRank by combining these two PageRanks. After having tested several possible combinations not discussed in this paper, we empirically chose to weight each node by the ratio of the two sample-based PageRank, after having set all null entries of the antiPageRank equal to the minimal non-null entry. 
Active-site first LiveRank (ASF) To compare with previous work, we propose the following variant inspired by the Dasgupta et al. [7] strategy for finding pages that have changed in a recrawl. Their algorithm is based on sampling for estimating page change rate for each website and then to crawl sites by decreasing change rate. In details, Active-site first (ASF) consists in partitioning $Z$ into websites determined by inspecting the URLs. We thus obtain a collection $Z_{1}, \ldots, Z_{p}$ of sets. For each set $Z_{i}$ corresponding to some site $i$, we obtain an estimation $\left|a\left(Z_{i}\right)\right| /\left|Z_{i}\right|$ of its activity (i.e. the fraction of active pages in the site). We then sort the remaining URLs by decreasing site activity.

\subsection{Dynamic LiveRanks}

Instead of using the acquired information just one time after the sampling, Dynamic LiveRanks are continuously computed and updated on the fly along the entire crawling process. On the one hand, this gives them real-time knowledge of $a$, but on the other hand, as the dynamic LiveRank may evolve all the time, they can create synchronization issues when used by distributed crawlers.

Like for sample-based LiveRanks, dynamic LiveRanks use a training set $Z$ of $z$ pages from a static LiveRank. This allows to bootstrap the adjustment by giving a non-empty knowledge of $a$, and prevents the LiveRank from focusing on only a small subset of $V$.

Breadth-First Search (BFS) With BFS, we aim at taking direct advantage of the possible propagation of liveness. The BFS queue is initialized with the (uncrawled) training set $Z$. The next page to be crawled is popped from the queue following First-In-First-Out (FIFO) rule. If the selected page appears to be alive, all of its uncrawled outgoing neighbors are pushed into the end of the queue. When the queue is empty, we pick the unvisited page with highest PageRank ${ }^{3}$.

Alive indegree (AI) BFS uses a simple FIFO queuing to determine the processing order. We now propose AI which provides a more advanced page selection scheme. For AI, each page in the graph is associated with a live score value indicating how many reported alive pages point to it. These values are set to zeros at the beginning and always kept up-to-date. AI is initialized by testing $Z$ : each node in $a(Z)$ will increment the associated values of its out-going neighbors by one. After $Z$ is tested, the next node to be crawled is simply the one with highest live score (in case of equality, to keep things consistent, we pick the node with highest PageRank). Whenever a new alive node is found, we update the live scores of its untested neighbors.

With Dynamic LiveRank, it is natural to think of a dynamic PageRank-based strategy where PageRank vector is recursively computed. Starting from a uniform distribution on $a(Z)$, we obtain $X$ in (1). Then a new teleportation vector is constructed as a uniform distribution on largest value entries of $X$, i.e., those which are considered probably alive after the first diffusion of $a(Z)$. The process continues and $X$ is updated iteratively. However, this method is not efficient since it can not escape from the locality of $a(Z)$.

\footnotetext{
${ }^{3}$ We tested several other natural options and observed no significant impact.
} 


\section{Datasets}

We chose to evaluate the proposed LiveRanks on datasets of the British domain .uk available on the WebGraph platform ${ }^{4}$. In this Section, we present these datasets, describe how we obtained the alive function $a$ and observe the correlations between $a$, indegree and PageRank.

\section{$3.1 \quad$ uk-2002 dataset}

The main dataset we will use is the web graph uk-2002 $2^{5}$ crawled by UbiCrawler [4]. This snapshot, crawled in 2002, contains 18,520,486 pages and 298,113,762 hyperlinks.

The preliminary task is to determine $a$, the liveness of the pages of the snapshot. For each URL, we have performed a GET request and hopefully obtained a corresponding HTTP code. Our main findings are:

- One third of the total pages are no longer available today, the server returns error 404.

- One fourth have a DNS problem (which probably means the website is also dead).

- For one fifth of the cases, the server sends back the redirection message 301. Most redirections for pages of an old site lead to the root of a new site. If we look at the proportion of distinct pages alive at the end of redirections, it is as low as $0.1 \%$.

- Less than $13 \%$ of pages return the code 200 (success). However, we found out that half of them actually display some text mentioning that the page was not found. To handle this issue, we have fully crawled all the pages with code 200 and filtered out pages whose title or content have either Page Not Found or Error 404.

The results are summarized in Table 1 . In the end, our methodology led to finding out 1,164,998 alive pages, accounting for $6.4 \%$ of the dataset.

\begin{tabular}{|c|c|c|c|}
\hline Status & Description & Number of pages & Percentage \\
\hline Code HTTP 404 & Page not found & 6467219 & $34,92 \%$ \\
\hline No answer & Host not found & 4470845 & $24,14 \%$ \\
\hline Code HTTP 301 & Redirection & 3455923 & $18,66 \%$ \\
\hline$-\overline{\text { Target }}-\overline{0} \overline{0}-$ & $\overline{\text { Target of }} \overline{\text { redirection }}$ & $-\overline{20} \overline{41} \overline{4}$ & $-\overline{0,1} \overline{1} \%$ \\
\hline Code HTTP 200 & Page exists & 2365201 & $12,77 \%$ \\
\hline${ }^{-}$True $\overline{200}-$ & Page really exists & $\overline{1} \overline{16} \overline{4} \overline{99}$ & $\overline{6,2} \overline{\mathbf{9}} \overline{\%}$ \\
\hline Others $(403, \ldots)$ & Other error & 1761298 & $9,51 \%$ \\
\hline Total & Graph size & 18520486 & $100 \%$ \\
\hline
\end{tabular}

Table 1: Status of web pages in uk-2002, crawled in December 2013.

\footnotetext{
${ }^{4}$ http://webgraph.di.unimi.it/

${ }^{5}$ http://law.di.unimi.it/webdata/uk-2002/
} 


\section{$3.2 \quad$ uk-2006 dataset}

The settings of uk-2002 are rather adversarial (old snapshot with relatively few alive pages), so we wanted to evaluate the impact of LiveRanks on shorter time scales. In absence of fresh enough available datasets, we used the DELIS dataset [5], a series of twelve continuous snapshots ${ }^{6}$ starting from $06 / 2006$ to $05 / 2007$ (one-month intervals). We set $G$ to the first snapshot $(06 / 2006)$. It contains $31,316,403$ nodes and 813,807,972 hyperlinks. We then considered the last snapshot $(05 / 2007)$ as "present time", setting the active set $a(V)$ as the intersection between the two snapshots. With this methodology, we hope to have a good approximation of $a$ after a one-year period. For this dataset, we obtained $n_{a}=11,142,177$ "alive" nodes representing $35.56 \%$ of the graph.

\subsection{Correlations}

The rationale behind the LiveRanks $I$ and $P$ is the assumption that the liveness of pages is correlated to the graph structure of the snapshot, so that a page with high in-degree or PageRank has more chances to stay alive.

To validate this, we plot in Figure 1 the cumulative distribution of in-degree (figure 1a) and PageRank (figure 1b) for alive, dead, and all pages of the uk-2002 dataset. We observe that the curve for active nodes is slightly shifted to the right compared to the other curves in each figures: active users tend to have slightly higher in-degree and PageRank than in the overall population. The bias is bigger for PageRank, suggesting that LiveRank $(\mathrm{P})$ should perform better than LiveRank (I).

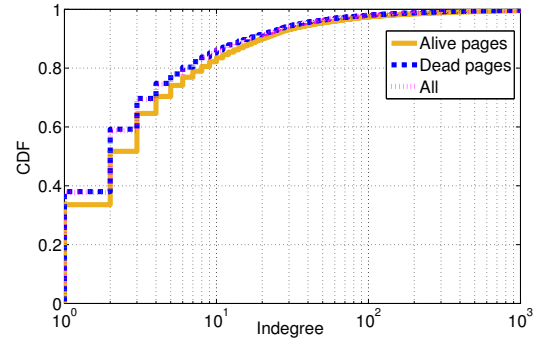

(a) uk-2002: Indegree CDF

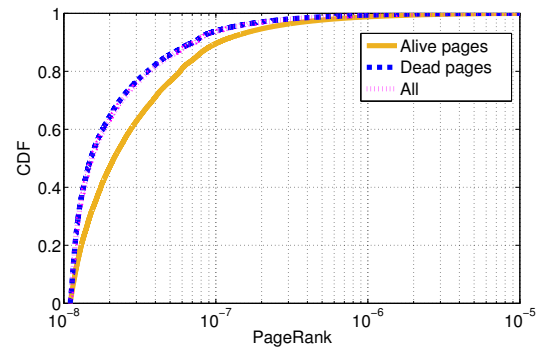

(b) uk-2002: PageRank CDF

Fig. 1: Cumulative distribution of pages according to Indegree and PageRank.

\section{LiveRanks evaluation}

After having proposed several LiveRanks in Section 2 and described our datasets in previous Section, we can now benchmark our proposals.

All our evaluations are based on representations of the cost functions. In each plot, the $\mathrm{x}$-axis indicates the fraction $\alpha$ of active nodes we aim to discover and the y-axis corresponds to the relative cost of the crawl required to achieve that goal. A low curve indicates an efficient LiveRank. Like said in Section 2.1: an ideal LiveRank would achieve a constant cost of 1; a random LiveRank is quickly

\footnotetext{
${ }^{6}$ http://law.di.unimi.it/webdata/uk-union-2006-06-2007-05/
} 
constant with an average cost $n / n_{a}$; any non-clairvoyant LiveRank will tend to cost $n / n_{a}$ as $\alpha$ goes to 1 .

We mainly focus on the uk-2002 dataset. When it is not specified, the training set contains the $z=100000$ pages of higher (static) PageRank.

\subsection{Static and sample-based LiveRanks}

We first evaluate the results of static and sample-based LiveRanks. The results are displayed in Figure 2. For static LiveRanks, we see as expected that a random ordering gives an almost constant cost equal to $\frac{n}{n_{a}} \approx 15.6$. Indegree ordering (I) and PageRank (P) significantly outperform this result, PageRank being the best of the three: it is twice more efficient than random for small $\alpha$, and still performs approximately $30 \%$ better when up to $\alpha=0.6$. We then notice that we can get even much better costs with sample-based approaches, the double-adaptive LiveRank $P_{a}^{+/-}$giving a significant improvement over the simple-adaptive one $P_{a} . P_{a}^{+/-}$allows improving the ordering by a factor of 6 approximately around $\alpha=0.2$ with a cost of 2.5 fetches per active node found. The cost for gathering half of the alive pages is less than 4, and for 90\% it stays less than 10 .

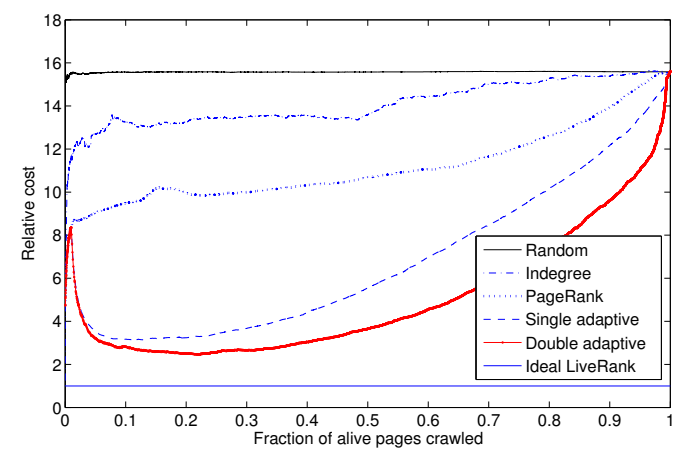

Fig. 2: Main results on static and sample-based LiveRanks

\subsection{Quantitative and qualitative impact of the training set}

We study in Figure 3 the impact of the training sets on sample-based LiveRanks. Results are shown for $P_{a}^{+/-}$but similar results were obtained for $P_{a}$.

Figure 3a shows the impact of the size $z$ of the sampling set (sampling the top PageRank pages). We observe some trade-off: as the sampling set grows larger, the initial cost increases as the sample does not used any fresh information, but it results in a significant increment of efficiency in the long run. For this dataset, taking a big training set $(z=500$ 000) allows reducing the cost of the crawl for $\alpha \geq 0.4$, and maintains a cost less than 4 for up to $90 \%$.

Another key aspect of the sampling phase is the qualitative choice of the sample set. Using $z=100$ 000, we can observe in Figure 3b that the performance of double adaptive $P_{a}^{+/-}$is further improved by using a random sample set 


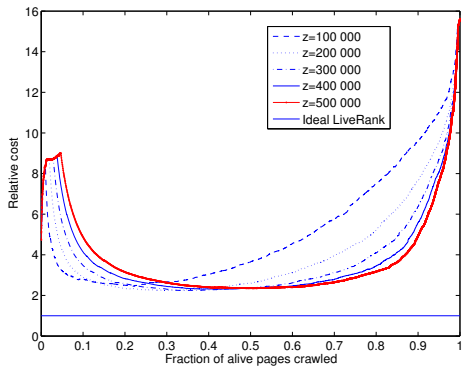

(a) Impact of the size $z$

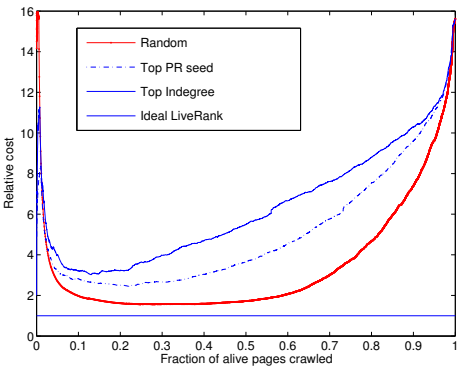

(b) Impact of the selection of $Z$

Fig. 3: Impact of the training set

rather than selecting it according to the PageRank or by decreasing indegree. We believe that the reason is that a random sample avoids a locality effect in the sampling set as high PageRank pages tend to concentrate in some local parts of the graph. To verify that, we tried to modify Indegree and PageRank selection to avoid to select neighbor pages. The results (not displayed here) show a significant improvement while staying less efficient than using a random sample.

Note that double-adaptive LiveRank through random sampling offers a very low cost, within a factor of 2 from optimal for a large range of values $\alpha$.

\subsection{Dynamic LiveRanks}

We then consider the performance of fully dynamic strategies, using the doubleadaptive LiveRank with random training set as a landmark. The results are displayed in Figure 4a. We see that bread-first search BFS and alive indegree AI perform similarly to double adaptive $P_{a}^{+/-}$for low $\alpha$ and can outperform it for large $\alpha$ (especially BFS). BFS begin to significantly outperform double adaptive for $\alpha \geq 0.5$. However, if one needs to gather half of the active pages or less, double adaptive is still the best candidate as it is much simpler to operate, especially with a distributed crawler.

Additionally, Figure 4b shows the impact of different sampling sets on BFS and AI. Except for high values of $\alpha$ where a random sampling outperforms other strategies, the type of sampling does not seem to affect the two dynamic LiveRanks as much as it was observed for the double-adaptive LiveRank.
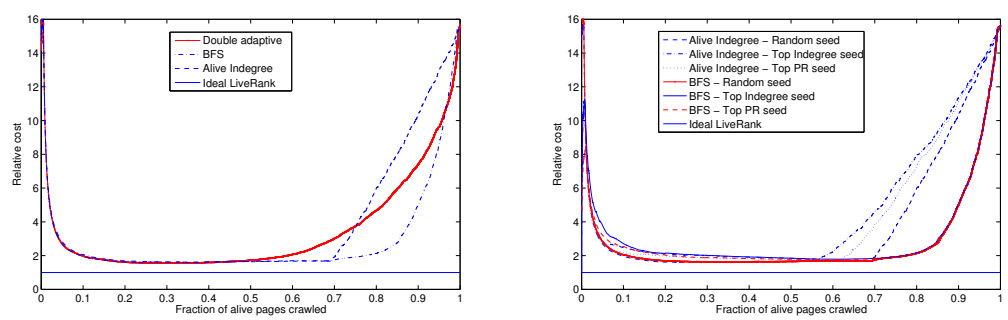

(a) Performance of dynamic LiveRanks(b) Impact of $Z$ on dynamic LiveRanks

Fig. 4: uk-2002 Performance of dynamic LiveRanks 


\section{4 uk-2006 dataset}

We have repeated the same experiments on the dataset uk-2006, where the update interval is only one year. We show in Figure 5 the results for static and sample-based LiveRanks, using for training set $z=200000$ (because the dataset is larger) and random sampling. The observation are qualitatively quite similar compared to uk-2002. The main difference is that all costs are lower due to a higher proportion of alive pages $\left(\frac{n}{n_{a}} \approx 2.81\right)$. The double-adaptive version still gives the lower relative cost among static and sample-based LiveRanks, staying under 1.4 for a wide range of $\alpha$.

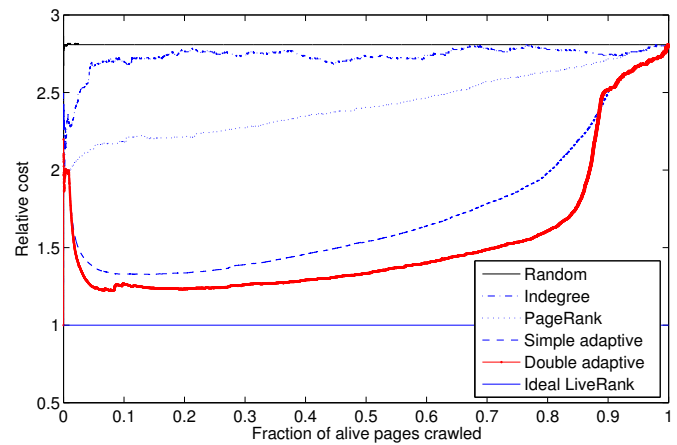

Fig. 5: uk-2006 main evaluation results

\subsection{Comparison with a site-based approach}

To compare with techniques from previous work for finding web pages that been updated after a crawl, Figure 6 compares double adaptive $P_{a}^{+/-}$to active-site first ASF with random sampling. The same number of random pages is tested in each site and the overall number of tests is the same as with double adaptive. Note that given the budget $z$, it was not possible to sample small websites. Unsampled websites are crawled after the sampled ones.

We see that for $\alpha$ greater than 0.9, ASF performs like a random LiveRank. This corresponds to the point where all sampled website have been crawled. That effect aside, the performance of ASF is not as good as double-adaptive LiveRank for earlier $\alpha$. In the end, ASF only beats $P_{a}^{+/-}$for a small range of $\alpha$, between 0.7 and 0.85 , and the gain within that range stays limited.

\section{Conclusion}

In this paper, we investigated how to efficiently retrieve large portions of alive pages from an old crawl using orderings we called LiveRanks. 


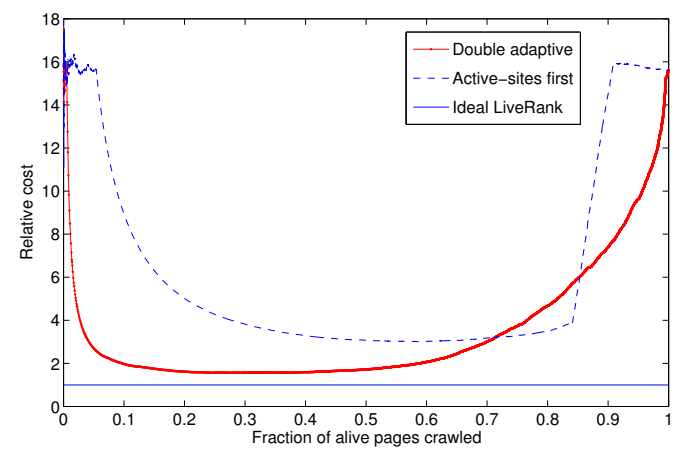

Fig. 6: Comparison with the cost of an active-site first LiveRank

We observed that PageRank is a good static LiveRank. However, we get a significant gain by first testing a small fraction of the pages to adjust the PageRank in a sample-based approach.

Compared to previous work on identifying modified pages, our technique performs similarly for a given large desired fraction (around 80\%) when compared to the LiveRank algorithm inspired by the technique in [6]. However, outside that range, our method outperforms this technique. Interesting future work could reside in using our techniques for the problem exposed in [6] (identification of pages that have changed) and compare with the Website sampling approach.

Interestingly, we could not get significant gain when using fully dynamic LiveRanks. As noticed before, each of the two phases of the sample-based approach can be easily parallelized through multiple crawlers whereas this would be much more difficult with a fully dynamic approach. The sample-based method could for example be implemented with in two rounds of a simple map-reduce program whereas the dynamic approach requires continuous exchanges of messages between the crawlers.

Our work establishes the possibility of efficiently recovering a significant portion of the alive pages of an old snapshot and advocates for the use of an adaptive sample-based PageRank for obtaining an efficient LiveRank.

To conclude, we emphasize that the LiveRank approach proposed in this paper is very generic, and its field of applications is not limited to Web graphs. It can be straightforwardly adapted to any online data with similar linkage enabling crawling, like P2P networks or online social networks. For future work, our approach will be mathematically extended. The problem can be formulated as an accuracy estimation of LiveRank vector, given a uniform distribution on the alive training set as teleportation vector. 


\section{References}

1. S. Abiteboul, M. Preda, and G. Cobena. Adaptive on-line page importance computation. In $W W W$ '03, pages 280-290. ACM, 2003.

2. Z. Bar-Yossef, A. Z. Broder, R. Kumar, and A. Tomkins. Sic transit gloria telae: Towards an understanding of the web's decay. In Proceedings of the 13th International Conference on World Wide Web (WWW'04), pages 328-337, 2004.

3. M. Bianchini, M. Gori, and F. Scarselli. Inside pagerank. ACM Trans. Internet Technol., 5(1):92-128, Feb. 2005.

4. P. Boldi, B. Codenotti, M. Santini, and S. Vigna. Ubicrawler: A scalable fully distributed web crawler. Software: Practice \& Experience, 34(8):711-726, 2004.

5. P. Boldi, M. Santini, and S. Vigna. A large time-aware graph. SIGIR Forum, 42(2):33-38, 2008.

6. J. Cho and A. Ntoulas. Effective change detection using sampling. In Proceedings of the 28th international conference on Very Large Data Bases, pages 514-525. VLDB Endowment, 2002.

7. A. Dasgupta, A. Ghosh, R. Kumar, C. Olston, S. Pandey, and A. Tomkins. The discoverability of the web. In $W W W$ '07, pages 421-430. ACM, 2007.

8. N. Eiron, K. S. McCurley, and J. A. Tomlin. Ranking the web frontier. In Proceedings of the 13th international conference on World Wide Web, pages 309-318. ACM, 2004.

9. T. Haveliwala, A. Kamvar, D. Klein, C. Manning, and G. Golub. Computing pagerank using power extrapolation. Technical report, 2003.

10. S. D. Kamvar, T. H. Haveliwala, C. D. Manning, and G. H. Golub. Extrapolation methods for accelerating pagerank computations. In $W W W$ '03, pages 261-270. ACM, 2003.

11. A. N. Langville and C. D. Meyer. Deeper inside pagerank. Internet Mathematics, $1: 2004,2004$.

12. C. Olston and M. Najork. Web crawling. Foundations and Trends in Information Retrieval, 4(3):175-246, 2010.

13. L. Page, S. Brin, R. Motwani, and T. Winograd. In The PageRank Citation Ranking: Bringing Order to the Web., number 1999-66. Stanford InfoLab, November 1999.

14. K. Radinsky and P. N. Bennett. Predicting content change on the web. In Proceedings of the sixth ACM international conference on Web search and data mining, pages 415-424. ACM, 2013.

15. Q. Tan, Z. Zhuang, P. Mitra, and C. L. Giles. A clustering-based sampling approach for refreshing search engine's database. In Proceedings of the 10th International Workshop on the Web and Databases, 2007. 